\title{
Treatment Approach for Knee Osteoarthritis with Ipsilateral Femoral Cartilage Tumor: A Case Series
}

\author{
Aadit Shah \\ Eric Lepkowsky \\ Alexander Duke \\ Meghan Moriarty \\ Haley Riordan \\ Fazel Khan
}

Stony Brook University Hospital, Department of Orthopaedics, Stony Brook, NY, I I794, USA
Correspondence: Aadit Shah

Stony Brook University Hospital,

Department of Orthopaedics, I0I Nicolls

Road, HSC T-I8 - 089, Stony Brook, NY,

11794, USA

Tel + |-63I-786-9484

Email Aadit.Shah@stonybrookmedicine.

edu
Abstract: Osteoarthritis (OA) is a common pathology affecting the knee joint. Twenty percent of the cartilaginous tumors of bone are in the distal femur. This presents a challenge for treating patients with knee OA who also have ipsilateral distal femur cartilage tumors. We propose a classification system for knee OA with ipsilateral cartilaginous tumor and a treatment approach to address this issue. Intramedullary guides are avoided when performing total knee arthroplasty (TKA) in these patients so as not to further contaminate the femur with tumor cells. A non-image-based stereotactic surgical system is favored at our institution to achieve this goal. Seven patients underwent classification and treatment with TKA utilizing this approach. Average final follow-up was 15.3 months. Component alignment averaged $89.2^{\circ}$ and all patients had $0^{\circ}$ of extension with an average flexion to $107.5^{\circ}$. There were no postoperative complications and no radiographic evidence of component complication, tumor recurrence, or tumor expansion. Few studies have reported on the treatment of knee OA with concurrent cartilaginous tumor of the distal femur. All patients treated with the proposed approach had their OA successfully treated without complication related to their cartilaginous tumor or TKA components. This case series presents a novel classification and treatment algorithm to potentially guide arthroplasty surgeons in approaching these often-concurrent occurring pathologies.

Keywords: total knee arthroplasty, osteoarthritis, cartilage tumor, enchondroma, chondrosarcoma

\section{Introduction}

Osteoarthritis (OA) of the knee is a significant health problem with an increasing percentage of patients in the United States (US) developing knee OA. ${ }^{1}$ Disability, pain, stiffness, and cost of treatment all contribute to the burden knee OA places on the healthcare system. ${ }^{2}$ Many symptomatic patients fail non-operative management and require surgical intervention, with favorable results seen in patients who undergo total knee arthroplasty (TKA). ${ }^{3}$

Cartilaginous tumors are common neoplasms involving bone that range from benign latent enchondromas to malignant high-grade chondrosarcomas, with many being found incidentally. ${ }^{4-6}$ Almost $20 \%$ of these tumors present in the distal femur. ${ }^{5}$ Differentiating an enchondroma from a low-grade chondrosarcoma is difficult because of the similar clinical and radiologic features. ${ }^{7}$ This presents another challenge as enchondromas are typically observed while low-grade chondrosarcomas undergo intralesional curettage with adjuvant therapy treatment. ${ }^{7}$

$\mathrm{OA}$ and cartilaginous tumors are common pathologies that can occur concurrently, presenting a unique challenge for arthroplasty surgeons. The following case 
series presents our approach for treating OA of the knee in patients with cartilaginous tumors of the ipsilateral distal femur as well as a new classification system that may help guide surgeons encountering this scenario.

\section{Case Reports}

Our institution developed a classification system for knee $\mathrm{OA}$ in patients with cartilaginous tumors of the ipsilateral femur (Table 1). Plain radiographs was the primary modality that was initially used to classify the lesion in one of the four types. Enchondromas and low-grade chondrosarcoma can have similar radiographic appearance. The

Table I Classification for Knee OA with Ipsilateral Cartilaginous Tumor and Treatment Recommendations

\begin{tabular}{|c|c|c|}
\hline $\begin{array}{l}\text { Classification } \\
\text { Type }\end{array}$ & Definition & $\begin{array}{l}\text { Treatment } \\
\text { Recommendation }\end{array}$ \\
\hline Type I & $\begin{array}{l}\text { Lesion suspicious for } \\
\text { enchondroma that is } \\
\text { remote from planned } \\
\text { distal femoral } \\
\text { component placement, } \\
\text { but in way of } \\
\text { intramedullary guide }\end{array}$ & $\begin{array}{l}\text { Perform TKA with } \\
\text { guides that avoid } \\
\text { intramedullary } \\
\text { contamination and } \\
\text { perform routine } \\
\text { observation of cartilage } \\
\text { lesion }\end{array}$ \\
\hline Type 2 & $\begin{array}{l}\text { Lesion suspicious for } \\
\text { enchondroma that is at } \\
\text { the level of planned } \\
\text { distal femoral } \\
\text { component placement }\end{array}$ & $\begin{array}{l}\text { Perform TKA with } \\
\text { guides that avoid } \\
\text { intramedullary } \\
\text { contamination and } \\
\text { perform concomitant } \\
\text { curettage of lesion with } \\
\text { augmentation for } \\
\text { arthroplasty as needed }\end{array}$ \\
\hline Type 3 & $\begin{array}{l}\text { Lesion suspicious for } \\
\text { low-grade } \\
\text { chondrosarcoma }\end{array}$ & $\begin{array}{l}\text { Perform intralesional } \\
\text { curettage with adjuvant } \\
\text { therapy, internal } \\
\text { fixation as needed; } \\
\text { then return to perform } \\
\text { TKA in a staged fashion } \\
\text { using appropriate } \\
\text { techniques to deal with } \\
\text { adjacent hardware }\end{array}$ \\
\hline Type 4 & $\begin{array}{l}\text { Lesion suspicious for } \\
\text { intermediate- to high- } \\
\text { grade chondrosarcoma }\end{array}$ & $\begin{array}{l}\text { Wide resection of } \\
\text { chondrosarcoma and, } \\
\text { when tumor is in the } \\
\text { vicinity of knee joint, } \\
\text { consider tumor } \\
\text { prosthesis that also } \\
\text { functions as total knee } \\
\text { arthroplasty }\end{array}$ \\
\hline
\end{tabular}

extent and degree of endosteal scalloping correlate the closest with the lesion being a chondrosarcoma. Murphy et $\mathrm{al}^{8}$ in their study found that $71(75 \%)$ of 95 patients with chondrosarcoma had endosteal scalloping of more than two-thirds of the cortical thickness, compared with 8 ( $9 \%$ ) of 92 patients with enchondroma. Based on this, the patients were classified in one of the four types in our classification system. Patients suspected to have chondrosarcoma based on the radiographs were then sent for biopsy to confirm the diagnosis and determine the grade of the tumor. This classification has guided the treatment of seven patients' osteoarthritis since its implementation in 2017. All TKAs were performed without intramedullary guides to avoid further contaminating the femur with pathologic cells. A medial parapatellar arthrotomy, cemented posterior stabilizing components, and patellar resurfacing were utilized for six of the seven cases. The last case is a case of intermediate chondrosarcoma where wide resection along with total femur replacement was performed.

Patients 1, 2, 3, and 4 were a 72-year-old female, a 77year-old male, a 73-year-old female, and an 83-year-old female, respectively. These patients had knee OA with incidentally found ipsilateral femoral enchondromas remote from planned distal femoral component. All four patients with remote ipsilateral femoral enchondroma will be Type 1 in our classification scheme. All four patients underwent TKA as described above (Figure 1A). Followup was 20 months, 14 months, 7 months, and 18 months for patients $1,2,3$, and 4, respectively, without postoperative complication. All four patients were observed with routine $\mathrm{X}$-rays with regards to their cartilage lesions, and all were noted to be stable.

Patient 5 was a 76-year-old female with knee OA and suspected ipsilateral femoral enchondroma that would be encountered during distal femur cuts. This patient will be Type 2 in our classification scheme. TKA was performed as described above and, when the tumor was encountered, curettage of the lesion was performed with argon beam as adjuvant therapy. Autologous bone graft from the tibial cuts was used to fill the void and a stemmed femoral implant was required to compensate for the bone loss (Figure 1B). This patient had 14 months of follow-up without complication or noted recurrence of her femoral lesion on routine imaging.

Patient 6 was an 85-year-old male with knee OA and an ipsilateral femur tumor concerning for low-grade chondrosarcoma. This patient will be Type 3 in our classification 
A

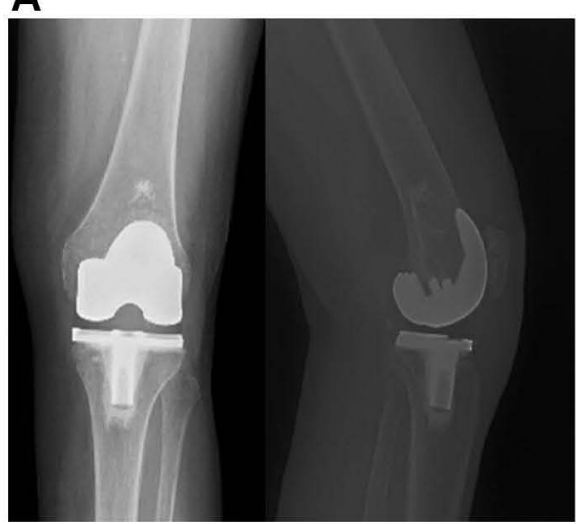

B

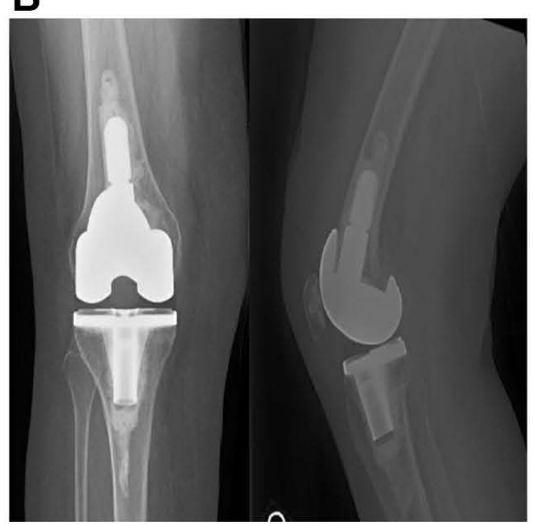

C

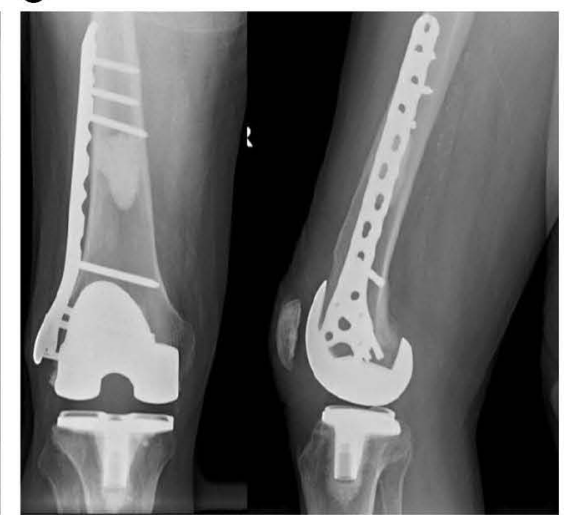

Figure I Post-operative X-rays from case report patients I-6 (A) AP and lateral of knee for Patient I with TKA and no additional treatment required for cartilaginous tumor. (B) AP and lateral of knee for Patient 5 with TKA and curettage with cement augmentation required for cartilaginous tumor. (C) AP and lateral of knee for Patient 6 who underwent curettage with cement augmentation and internal fixation of cartilaginous tumor prior to treatment of OA with a TKA.

scheme. Intralesional curettage with calcium phosphate grafting and prophylactic fixation with a plate and screw construct was performed. High-grade chondrosarcoma was ruled out by biopsy at time of surgery. Patient 6 returned at a later date for TKA performed using the above steps, as now the calcium phosphate and hardware prevented placement of an intramedullary guide (Figure 1C). This patient had 25 months of follow-up without complication or noted recurrence of his femoral lesion on routine imaging.

Patient 7 was a 63-year-old male with knee and ipsilateral thigh pain who was found to have a lesion of the femur concerning for intermediate to high-grade chondrosarcoma. This patient will be Type 4 in our classification scheme. A biopsy of the lesion was performed which revealed intermediate-grade chondrosarcoma. For this patient, wide resection of the tumor along with total femur replacement was performed (Figure 2). This patient had nine months of follow-up without complication or any evidence of recurrence or metastasis.

\section{Discussion}

Osteoarthritis (OA) is a common pathology that impacts the knee joint and up to $20 \%$ of low-grade cartilaginous tumors can occur within the distal femur. ${ }^{4,5}$ Over the last several decades, chondrogenic tumors of the appendicular skeleton, other than those of hands and feet, have been known as a diagnostic dilemma in literature. ${ }^{9}$ It is even more challenging if the lesion lies close to another musculoskeletal pathology such as OA. If no concomitant OA was present, current literature would propose observation of enchondromas, intralesional treatment for a low-grade cartilaginous tumor, and wide resection for intermediate and high-grade chondrosarcomas. ${ }^{10,11}$ This case series presents an approach used at our institution for performing TKA in patients with
A

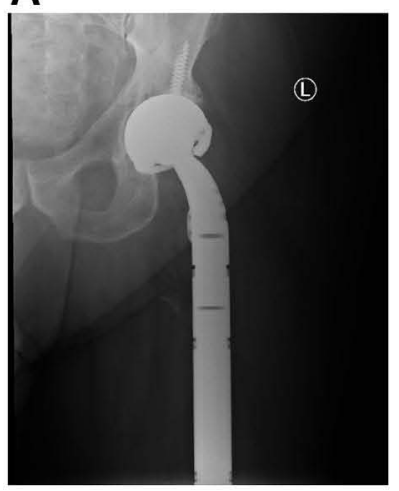

B

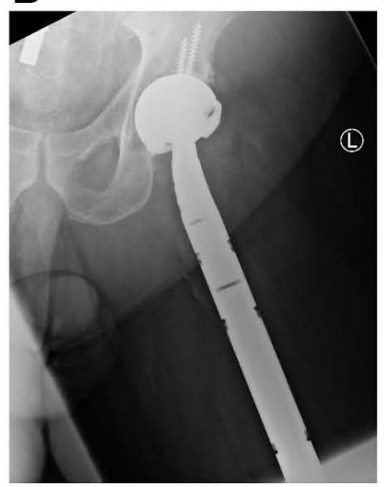

C

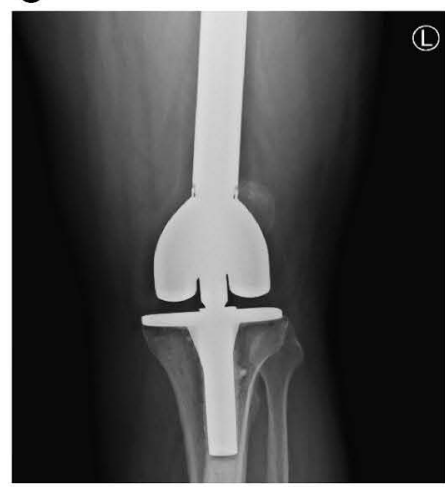

D

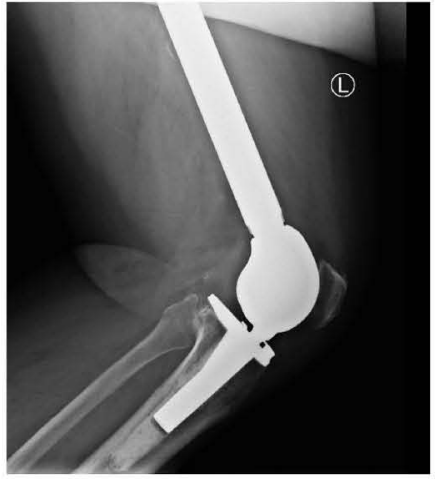

Figure 2 Post- operative X-ray from case report Patient 7 who underwent wide resection and total femur replacement. (A) AP hip, (B) lateral hip, (C) Ap knee, (D) lateral knee. 
knee OA and cartilaginous tumors of the ipsilateral distal femur.

All seven of our patients had end-stage OA that failed conservative treatment, and thus were deemed to require operative treatment with TKA. To date, there is only one case study, of two patients, regarding the treatment of knee OA with an ipsilateral, periarticular cartilage tumor. ${ }^{12}$ However, this study utilized intramedullary guides for their patients as well as being unable to delineate treatment based on tumor grade due to their sample size. ${ }^{12} \mathrm{We}$ sought to avoid intramedullary guides in order to minimize the chance of seeding the remaining bone with tumor cells. One additional study was found in the literature search that reported on this clinical scenario. No previous report detailing a treatment algorithm for knee OA with ipsilateral femoral cartilage lesions was found in the literature.

For all seven patients included in this case series, radiographs as well as clinical and physical exams were conducted during their postoperative visit. Radiographs were all reviewed by a blinded radiologist. Average alignment of the components was measured to be $89.2^{\circ}$, with a range of $88.0^{\circ}$ to $90.0^{\circ}$. In addition, there was no radiographic evidence of fracture, aseptic loosening, or hardware complications. Neither patient who had undergone curettage and cement augmentation demonstrated recurrence. The patient who underwent wide resection did not demonstrate any recurrence or metastasis. The remaining patients had no radiographic changes in their cartilage tumors on subsequent imaging studies. All patients also had their range of motion (ROM) determined at each postoperative visit, with all patients achieving $0^{\circ}$ of extension and average flexion to $107.5^{\circ}$ with a range of $100^{\circ}$ to $125^{\circ}$.

The classification and recommendations we propose for patients with knee OA and ipsilateral femoral cartilage tumors are summarized in Table 1. We propose avoiding intramedullary guides so as not to contaminate the femur further with pathologic cells for Type 1 and 2 lesions. Adjuvant treatment for Type 2 and 3 lesions can be determined by surgeon preference, with ours being argon beam therapy and internal fixation as needed. We additionally propose that Type 3 lesions be approached in a staged fashion. For a Type 4 lesion, which is an intermediateor high-grade cartilaginous tumor, our recommendation would be to perform a wide resection along with a prosthesis that also functions as a total knee arthroplasty such as a total or distal femoral replacement. This case series serves as a pilot study with potential for future studies to be conducted to confirm the results presented here.

\section{Ethical Approval}

The case series was authorized by Stony Brook University Institutional Review Board. This study was conducted in accordance with the Declaration of Helsinki.

\section{Consent for Publication}

All patients were aware of their participation in the case series and informed consent was obtained from all patients/next of kin for publication.

\section{Acknowledgments}

No support was received for this paper. The investigation was performed at Stony Brook University Hospital, Stony Brook, New York, USA.

\section{Disclosure}

Dr Fazel Khan reports fees from NaviSect during the conduct of the study and fees from NaviSect outside the submitted work. In addition, Dr Fazel Khan has a patent, US2020/0360093 A1, pending. The authors declare no other potential conflicts of interest.

\section{References}

1. Centers for Disease Control and Prevention (CDC). Projected statespecific increases in self-reported doctor-diagnosed arthritis and arthritis-attributable activity limitations-United States, 2005-2030. MMWR Morb Mortal Wkly Rep. 2007;56(17):423-425.

2. Kurtz S, Ong K, Lau E, Mowat F, Halpern M. Projections of primary and revision hip and knee arthroplasty in the United States from 2005 to 2030. J Bone Joint Surg Am. 2007;89(4):780-785. doi:10.2106/ JBJS.F.00222

3. Núñez M, Lozano L, Núñez E, et al. Total knee replacement and health-related quality of life: factors influencing long-term outcomes. Arthritis Rheum. 2009;61(8):1062-1069. doi:10.1002/art.24644

4. Unni KK. Cartilaginous lesions of bone. J Orthop Sci. 2001;6 (5):457-472. doi:10.1007/s007760170015

5. Whyte M. Acquired Disorders of Cartilage and Bone. American Society for Bone and Mineral Research: Washington DC, USA; 2003.

6. Mulligan ME. How to diagnose enchondroma, bone infarct, and chondrosarcoma. Curr Probl Diagn Radiol. 2019;48(3):262-273. doi:10.1067/j.cpradiol.2018.04.002

7. Deckers C, Schreuder BH, Hannink G, de Rooy JW, van der Geest IC. Radiologic follow-up of untreated enchondroma and atypical cartilaginous tumors in the long bones. J Surg Oncol. 2016;114(8):987-991. doi: $10.1002 /$ jso. 24465

8. Murphey MD, Andrews CL, Flemming DJ, Temple HT, Smith WS, Smirniotopoulos JG. From the archives of the AFIP. Primary tumors of the spine: radiologic pathologic correlation. Radiographics. 1996;16 (5):1131-1158. doi:10.1148/radiographics.16.5.8888395

9. Murphey MD, Flemming DJ, Boyea SR, Bojescul JA, Sweet DE, Temple HT. Enchondroma versus chondrosarcoma in the appendicular skeleton: differentiating features. Radiographics. 1998;18 (5):1213-1245. doi:10.1148/radiographics.18.5.9747616 
10. Chen X, Yu LJ, Peng HM, et al. Is intralesional resection suitable for central grade 1 chondrosarcoma: a systematic review and updated meta-analysis. Eur $J$ Surg Oncol. 2017;43(9):1718-1726. doi:10.1016/j.ejso.2017.05.022

11. Omlor GW, Lohnherr V, Hetto P, et al. Surgical therapy of benign and low-grade malignant intramedullary chondroid lesions of the distal femur: intralesional resection and bone cement filling with or without osteosynthesis. Strateg Trauma Limb Reconstr. 2018;13(3):163-170. doi:10.1007/s11751-018-0321-2
12. Mortazavi SMJ, Razzaghof M, Moharrami A, Shamabadi A, Noori A. Total knee arthroplasty and atypical cartilaginous tumor/ enchondroma of the distal femur. Arthroplast Today. 2020;6 (3):521-525. doi:10.1016/j.artd.2020.06.005

\section{Publish your work in this journal}

Orthopedic Research and Reviews is an international, peer-reviewed, open access journal that focusing on the patho-physiology of the musculoskeletal system, trauma, surgery and other corrective interventions to restore mobility and function. Advances in new technologies, materials, techniques and pharmacological agents are particularly welcome. The manuscript management system is completely online and includes a very quick and fair peer-review system, which is all easy to use. Visit http://www.dovepress.com/testimonials.php to read real quotes from published authors. 